ALEA, Lat. Am. J. Probab. Math. Stat. 17, 473-489 (2020)

DOI: 10.30757/ALEA.v17-19

\title{
Localisation in a growth model with interaction. Arbitrary graphs
}

\author{
Mikhail Menshikov and Vadim Shcherbakov \\ Affiliation of Mikhail Menshikov \\ Department of Mathematical Sciences, \\ Durham University, \\ Durham, UK. \\ E-mail address: mikhail.menshikov@durham.ac.uk \\ Affiliation of Vadim Shcherbakov \\ Department of Mathematics, \\ Royal Holloway University of London, \\ Egham, UK. \\ E-mail address: vadim.shcherbakov@rhul.ac.uk
}

Abstract. This paper concerns the long term behaviour of a growth model describing a random sequential allocation of particles on a finite graph. The probability of allocating a particle at a vertex is proportional to a log-linear function of numbers of existing particles in a neighbourhood of a vertex. When this function depends only on the number of particles in the vertex, the model becomes a special case of the generalised Pólya urn model. In this special case all but finitely many particles are allocated at a single random vertex almost surely. In our model interaction leads to the fact that, with probability one, all but finitely many particles are allocated at vertices of a maximal clique.

\section{The model and main results}

Let $G=(V, E)$ be a non-oriented, finite connected graph with vertex set $V$ and edge set $E$. We write $v \sim u$ to denote that vertices $v$ and $u$ are adjacent, and $v \nsim u$, if they are not. By convention, $v \nsim v$ for all $v \in V$. Let $\mathbb{Z}_{+}$be the set of all nonnegative integers and let $\mathbb{R}$ be the set of real numbers. Given $\mathbf{x}=\left(x_{v}, v \in V\right) \in \mathbb{Z}_{+}^{V}$ define the growth rates as

$$
\Gamma_{v}(\mathbf{x}):=e^{\alpha x_{v}+\beta \sum_{u \sim v} x_{u}}, v \in V,
$$

Received by the editors July 3rd, 2019; accepted April 9th, 2020.

2010 Mathematics Subject Classification. 60K35, 60J10.

Key words and phrases. Growth Process, Cooperative Sequential Adsorption, Urn Models, Graph Based Interaction, Maximal Clique. 
where $\alpha, \beta \in \mathbb{R}$ are two given constants. Consider a discrete-time Markov chain $X(n)=\left(X_{v}(n), v \in V\right) \in \mathbb{Z}_{+}^{V}$ with the following transition probabilities

$$
\begin{aligned}
\mathrm{P}\left(X(n+1)=X(n)+\mathbf{e}_{v} \mid X(n)=\mathbf{x}\right) & =\frac{\Gamma_{v}(\mathbf{x})}{\Gamma(\mathbf{x})}, \quad \mathbf{x} \in \mathbb{Z}_{+}^{V}, \quad v \in V, \\
\Gamma(\mathbf{x}) & =\sum_{v \in V} \Gamma_{v}(\mathbf{x}),
\end{aligned}
$$

where $\mathbf{e}_{v} \in \mathbb{Z}_{+}^{V}$ is the $v$-th unit vector and $\Gamma_{v}(\mathbf{x})$ is defined in (1.1).

Definition 1.1. The Markov chain $X(n)=\left(X_{v}(n), v \in V\right) \in \mathbb{Z}_{+}^{V}$ with transition probabilities (1.2) is called the growth process with parameters $(\alpha, \beta)$ on the graph $G=(V, E)$.

The growth process $X(n)=\left(X_{v}(n), v \in V\right)$ describes a random sequential allocation of particles on the graph, where $X_{v}(n)$ is interpreted as the number of particles at vertex $v$ at time $n$. The growth process can be regarded as a particular variant of an interacting urn model on a graph. The latter is a probabilistic model obtained from an urn model by adding graph based interaction (e.g., Benaïm et al., 2015 and Fleurke et al., 2011). The growth process is motivated by cooperative sequential adsorption model (CSA). CSA is widely used in physics and chemistry for modelling various adsorption processes (Evans, 1993). The main peculiarity of adsorption processes is that adsorbed particles can change adsorption properties of the material. For instance, the subsequent particles might be more likely adsorbed around the locations of previously adsorbed particles. In this paper we study the long term behaviour of the growth process with positive parameters $\alpha$ and $\beta$. Positive parameters generate strong interaction so that existing particles increase the growth rates in the neighbourhood of their locations. This results in that, with probability one, all but finitely many particles are allocated at vertices of a maximal clique (see Definition 1.2 below). In a sense, the localisation effect is similar to localisation phenomena observed in other random processes with reinforcement (e.g. Basdevant et al., 2014 and Volkov, 2001).

The growth rates defined in equation (1.1) can be generalised as follows

$$
\Gamma_{v}(\mathbf{x})=e^{\alpha_{v} x_{v}+\sum_{u \sim v} \beta_{v u} x_{u}}, \quad v \in V, \quad \mathbf{x}=\left(x_{u}, u \in V\right),
$$

where $\left(\alpha_{v}, v \in V\right)$ and $\left(\beta_{v u}, v, u \in V\right)$ are arrays of real numbers. Setting $\alpha_{v} \equiv \alpha$, $\beta_{v u} \equiv \beta$ gives the growth process defined in Definition 1.1. Originally, the growth process with parameters $\alpha_{v}=\beta_{v u} \equiv \lambda \in \mathbb{R}$ on a cycle graph $G$ was studied in Shcherbakov and Volkov (2010b). The limit cases of the model in Shcherbakov and Volkov (2010b) $(\lambda=\infty$ and $\lambda=-\infty$ with convention $\infty \cdot 0=0)$ were studied in Shcherbakov and Volkov (2010a). The growth process on a cycle graph $G$ and with growth rates given by (1.3), where $\alpha_{v}=\beta_{v u}=\lambda_{v}>0, v, u \in V$, was studied in Costa et al. (2018). Note that if $\beta=0$ in (1.1), then the growth process is a special case of the generalised Pólya urn model with exponential weights (see, e.g. Davis, 1990).

We need the following definitions from the graph theory.

Definition 1.2. Let $G=(V, E)$ be a finite graph.

1) Given a subset of vertices $V^{\prime} \subseteq V$ the corresponding induced subgraph is a graph $G^{\prime}=\left(V^{\prime}, E^{\prime}\right)$ whose edge set $E^{\prime}$ consists of all of the edges in $E$ 
that have both endpoints in $V^{\prime}$. The induced subgraph $G^{\prime}$ is also known as a subgraph induced by vertices $v \in V^{\prime}$.

2) A complete induced subgraph is called a clique. A maximal clique is a clique that is not an induced subgraph of another clique.

Theorem 1.3 below is the main result of the paper.

Theorem 1.3. Let $X(n)=\left(X_{v}(n), v \in V\right) \in \mathbb{Z}_{+}^{V}$ be a growth process with parameters $(\alpha, \beta)$ on a finite connected graph $G=(V, E)$ with at least two vertices and let $0<\alpha \leq \beta$. Then for every initial state $X(0)=\mathbf{x} \in \mathbb{Z}_{+}^{V}$ with probability one there exists a random maximal clique with a vertex set $U \subseteq V$ such that

$$
\begin{aligned}
& \lim _{n \rightarrow \infty} X_{v}(n)=\infty \text { if and only if } v \in U, \text { and } \\
& \lim _{n \rightarrow \infty} \frac{X_{v}(n)}{X_{u}(n)}=e^{C_{v u}}, \text { for } v, u \in U,
\end{aligned}
$$

where

$$
\begin{aligned}
& C_{v u}=\lambda \lim _{n \rightarrow \infty} \sum_{w \in V} X_{w}(n)\left[\mathbf{1}_{\{w \sim v, w \nsim u\}}-\mathbf{1}_{\{w \sim u, w \nsim v\}}\right], \text { if } 0<\lambda:=\alpha=\beta, \text { and } \\
& C_{v u}=0, \text { if } 0<\alpha<\beta .
\end{aligned}
$$

Remark 1.4. In other words, Theorem 1.3 states that, with probability one, starting from a finite random time moment all subsequent particles are allocated at a random maximal clique. This is what we call localisation of the growth process. Note that quantities $C_{v u}$ are random and depend on the state of the process at the time moment, when localisation starts at the maximal clique.

Example 1.5. In Figure 1.1 we provide an example of a connected graph, where a growth process with parameters $0<\alpha \leq \beta$ can localise in five possible ways. The graph has eight vertices labeled by numbers $1,2,3,4,5,6,7$ and 8 . There are five maximal cliques induced by vertex sets $\{1,2\},\{2,7\},\{4,8\},\{7,8\},\{4,5,6\}$ and $\{2,3,4,5\}$ respectively. By Theorem 1.3, a growth process with parameters $0<\alpha \leq \beta$ can localise at any of these maximal cliques with positive probability, and no other limiting behaviour is possible.

For completeness, we state and prove the following result concerning the limit behaviour of the growth process in the case $0<\beta<\alpha$.

Theorem 1.6. Let $X(n)=\left(X_{v}(n), v \in V\right) \in \mathbb{Z}_{+}^{V}$ be a growth process with parameters $(\alpha, \beta)$ on a finite connected graph $G=(V, E)$ and let $0<\beta<\alpha$. Then for every initial state $X(0)=\mathbf{x} \in \mathbb{Z}_{+}^{V}$ with probability one there exists a random vertex $v$ such that

$$
\lim _{n \rightarrow \infty} X_{u}(n)=\infty \text { if and only if } u=v .
$$

In other words, with probability one, all but a finite number of particles are allocated at a single random vertex.

Remark 1.7. It is noted above, that if $\beta=0$, i.e. in the absence of interaction, our model becomes a special case of the generalised Pólya urn model, where a particle is allocated at a vertex $v$ with probability proportional to $e^{\alpha x_{v}}$, if the process is at state $\mathbf{x}=\left(x_{v}, v \in V\right) \in \mathbb{Z}_{+}^{V}$. In this case all but a finite number of particles are allocated at a random single vertex with probability one, if $\alpha>0$. Note that this particular result follows from a well known more general result for the generalised 


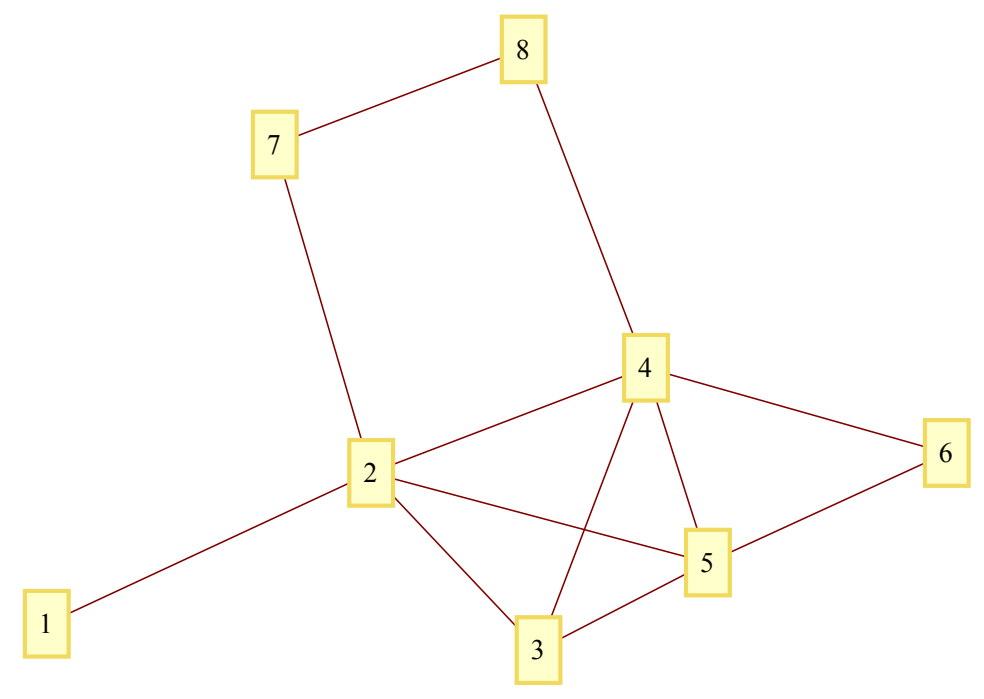

Figure 1.1. Graph with five cliques

Pólya urn model (Davis, 1990). The attractive interaction introduced in our model by a positive parameter $\beta$ leads to the fact that the growth process localises at a maximal clique rather than at a single vertex.

Remark 1.8. In Janson et al. (2019) and Shcherbakov and Volkov (2015) the long term behaviour of a continuous time Markov chain (CTMC) $\xi(t) \in \mathbb{Z}_{+}^{V}$, where $V$ is vertex set of a finite graph $G(V, E)$, was studied. Given state $\mathbf{x}=\xi(t) \in \mathbb{Z}_{+}^{V}$ a component $\xi_{v}(t)$ of the Markov chain increases by one with the rate equal to the growth rate $\Gamma_{v}(\mathbf{x})$ defined in (1.1), and a non-zero component decreases by one with the unit rate. Both papers Janson et al. (2019) and Shcherbakov and Volkov (2015) were mostly concerned with classification of the long term behaviour of the Markov chain, namely, whether the Markov chain is recurrent or transient depending on both the parameters $\alpha, \beta$ and the graph $G$. The typical asymptotic behaviour of the Markov chain was studied in Shcherbakov and Volkov (2015) in some transient cases. First of all, it was shown in Shcherbakov and Volkov (2015) that if both $\alpha>0$ and $\beta>0$, then, with probability one, there is a random finite time after which none of the components of CTMC $\xi(t)$ decreases. In other words, with probability one, the corresponding discrete time Markov chain (known also as the embedded Markov chain) asymptotically evolves as the growth process with parameters $(\alpha, \beta)$. Further, if $0<\beta<\alpha$, then, with probability one, a single component of CTMC $\xi(t)$ explodes. Theorem 1.6 above is basically the same result formulated in terms of the growth process. Another result of paper Shcherbakov and Volkov (2015) is that if $0<\alpha \leq \beta$ and the graph $G$ is connected, has at least two vertices and does not have cliques of size more than 2 , then, with probability one, only a pair of adjacent components of the Markov chain explodes. Theorem 1.3 in the present paper yields the following generalisation of this result on the case of arbitrary graphs. Namely, if $0<\alpha \leq \beta$, then, with probability one, only a group of CTMC $\xi(t)$ components labeled by vertices of a maximal clique explodes. 
Remark 1.9. Note also that in the case of a cycle graph and $\alpha=\beta>0$ localisation of the growth process at a pair of adjacent vertices was previously shown in Shcherbakov and Volkov (2010b, Theorem 3) and Costa et al. (2018, Theorem 1).

Let us briefly comment on proofs of Theorems 1.3 and 1.6. In both cases, given any initial state $X(0)=\mathrm{x}$ we identify special events that result in localisation of the growth process as described in the theorems. We show that the probability of any event of interest is bounded below uniformly over initial configurations. Then it follows from a renewal argument that almost surely one of these events eventually occurs. Note that the same renewal argument was used in Costa et al. (2018).

In the case of Theorem 1.6 we show by a direct computation that given any initial state $X(0)=\mathbf{x}$, with positive probability (depending only on the model parameters), all particles will be allocated at a single vertex with the maximal growth rate.

In the case of Theorem 1.3, we start with detecting a maximal clique, where the growth process can potentially localise. To this end, we use a special algorithm explained in Section 2.3. Given any initial state $X(0)=\mathrm{x}$ the algorithm outputs a maximal clique satisfying certain conditions (we call it final maximal clique, see Section 2.3). The key step in the proof is to obtain a uniform lower bound for the probability that all particles are allocated at vertices of a final maximal clique (Lemma 3.1 below). Given that all particles are allocated at vertices of a maximal clique we show that the pairwise ratios of numbers of allocated particles at the clique vertices converge, as claimed in Theorem 1.3. If $\alpha=\beta$, then convergence of the ratios follows from the strong law of large numbers for the i.i.d. case and a certain stochastic dominance argument. If $\alpha<\beta$, then for complete graphs convergence of the ratios follows from a strong law of large numbers for these graphs (Lemma 3.5). In the case $\alpha<\beta$ and arbitrary graphs the convergence of ratios follows from the result for complete graphs combined with the stochastic dominance argument.

The rest of the paper is organised as follows. In Section 2, we introduce notations and give definitions used in the proofs. The proof of Theorem 1.3 appears in Section 3, and Theorem 1.6 is proved in Section 4.

\section{Preliminaries}

2.1. Partition of the graph. Let $G=(V, E)$ be a finite connected graph with at least two vertices. Let $G\left(v_{1}, \ldots, v_{m}\right)$ denote a subgraph induced by vertices $v_{1}, \ldots, v_{m}$.

Definition 2.1. ( $D$-sets.) Let $\left(v_{1}, \ldots, v_{m}\right) \subseteq V$ be an ordered subset of vertices and let subgraph $G\left(v_{1}, \ldots, v_{m}\right)$ be a maximal clique. Define the following subsets of vertices $D_{v_{1}}, \ldots, D_{v_{m}}$

1) $D_{v_{1}}=\left\{v \in V: v \nsim v_{1}\right.$ and $\left.v \neq v_{1}\right\}$ and

2) $D_{v_{k}}=\left\{v \in V: v \nsim v_{k}, v \neq v_{k}\right.$ and $\left.v \sim v_{1}, \ldots, v_{k-1}\right\}$ for $2 \leq k \leq m$.

It follows from the definition of $D$-sets that

$$
\begin{aligned}
\left\{v_{1}, \ldots, v_{m}\right\} & \cap D_{v_{k}}=\emptyset, k=1, \ldots, m, \\
D_{v_{k}} & \cap D_{v_{j}}=\emptyset, v_{k} \neq v_{j} \text { for } v_{k}, v_{j} \in\left\{v_{1}, \ldots, v_{m}\right\}, \\
V & =\left\{v_{1}, \ldots, v_{m}\right\} \cup D_{v_{1}} \cup \ldots \cup D_{v_{m}} .
\end{aligned}
$$

Example 2.2. It should be noted that a $D$-set can be empty. For instance, let $G$ be the graph in Figure 1.1. Consider the clique with ordered set of vertices 
$\left(v_{1}=1, v_{2}=2\right)$, i.e. $G(1,2)$. Then $D_{v_{1}}:=D_{1}=\{3,4,5,6,7,8\}$ and $D_{v_{2}}:=D_{2}=\emptyset$. On the other hand, for the clique $G\left(v_{1}, v_{2}\right):=G(2,1)$, i.e. the clique with the reverse order of vertices, we have that $D_{v_{1}}=: D_{2}=\{6,8\}$ and $D_{v_{2}}:=D_{1}=\{3,5,4,7\}$.

2.2. Measure $\mathbf{Q}_{\mathbf{x}, n}$. In this section we introduce an auxiliary probability measure associated with the growth process. This measure naturally appears in the proof of Lemma 3.1 below and plays an important role in the proof.

Let $v_{1}, \ldots, v_{m}$ be an ordered set of vertices such that the induced graph $G\left(v_{1}, \ldots\right.$, $v_{m}$ ) is a maximal clique and let $D_{v_{1}}, \ldots, D_{v_{m}}$ be the corresponding $D$-sets. Define

$$
V_{k}:=\left\{v_{k}\right\} \cup D_{v_{k}}, k=1, \ldots, m .
$$

Given $i \in\{1, \ldots, m\}$ define the following events

$$
\begin{aligned}
& A_{n}^{v_{i}}=\left\{\text { at time } n \text { a particle is placed at site } v_{i}\right\}, n \geq 1, \\
& A_{n}^{V_{i}}=\left\{\text { at time } n \text { a particle is placed at site } v \in V_{i}\right\}, n \geq 1 .
\end{aligned}
$$

Let $\mathrm{P}_{\mathbf{x}}(\cdot)=\mathrm{P}(\cdot \mid X(0)=\mathbf{x})$ denote the distribution of the growth process started at $\mathbf{x} \in \mathbb{Z}_{+}^{V}$. Define the following set of vertex sequences

$$
S(n)=\{(k(1), \ldots, k(n)): k(i) \in(1, \ldots, m), i=1, \ldots, n\}, n \geq 1 .
$$

A sequence $(k(1), \ldots, k(n)) \in S(n)$ corresponds to an event, where a particle is allocated at vertex $v_{k(i)} \in\left(v_{1}, \ldots, v_{m}\right)$ at time $i, i=1, \ldots, n$.

Remark 2.3. Note that a sequence $\left(v_{k(1)}, \ldots, v_{k(n)}\right) \in S(n)$ uniquely determines a path $\mathbf{x}(1), \ldots, \mathbf{x}(n)$ of length $n$ of the growth process, where

$$
\mathbf{x}(j)=\mathbf{x}+\sum_{i=1}^{j} \mathbf{e}_{v_{k(i)}}, j=1, \ldots, n .
$$

It is easy to see that for each $\left(v_{k(1)}, \ldots, v_{k(n)}\right) \in S(n)$

$$
\mathrm{P}_{\mathbf{x}}\left(A_{j+1}^{V_{k(j+1)}} \mid \bigcap_{i=1}^{j} A_{i}^{v_{k(i)}}\right)=\mathrm{P}_{\mathbf{x}+\sum_{i=1}^{j} \mathbf{e}_{v_{k(i)}}}\left(A_{1}^{V_{k(j+1)}}\right), j=0, \ldots, n-1 .
$$

Let $\mathrm{Q}_{\mathbf{x}, n}$ denote a measure on $S(n)$ defined as follows

$$
\mathrm{Q}_{\mathbf{x}, n}\left(\left(v_{k(1)}, \ldots, v_{k(n)}\right)\right)=\mathrm{P}_{\mathbf{x}}\left(A_{1}^{V_{k(1)}}\right) \prod_{j=1}^{n-1} \mathrm{P}_{\mathbf{x}+\sum_{i=1}^{j} \mathbf{e}_{v_{k(i)}}}\left(A_{1}^{V_{k(j+1)}}\right) .
$$

It follows from equations (2.1)-(2.4) that $V_{k}, k=1, \ldots, m$, is a partition of the vertex set $V$ of the graph. In turn, this fact implies the following proposition.

Proposition 2.4. $\mathrm{Q}_{\mathbf{x}, n}$ is a probability measure on $S(n)$, that is

$$
\sum_{\left(v_{k(1)}, \ldots, v_{k(n)}\right) \in S(n)} \mathbf{Q}_{\mathbf{x}, n}\left(\left(v_{k(1)}, \ldots, v_{k(n)}\right)\right)=1,
$$

where the sum is taken over all elements of $S(n)$. 
2.3. Final maximal clique. For every initial state $X(0)=\mathbf{x}$ we detect a maximal clique, where the growth process can potentially localise, by using an algorithm described below. Denote for short $\Gamma_{v}=\Gamma_{v}(\mathbf{x}), v \in V$.

- Step 1. Let $v_{1}$ be a vertex such that $\Gamma_{v_{1}}=\max \left(\Gamma_{v}: v \in V\right)$. If there are several vertices with the maximal growth rate, then choose any of these vertices arbitrary.

- Step 2. Given vertex $v_{1}$ with the maximal growth rate, let $v_{2}$ be a vertex such that $\Gamma_{v_{2}}=\max \left(\Gamma_{v}: v \sim v_{1}\right)$. If there is more than one such vertex, then choose any of them arbitrarily. By construction, a subgraph $G\left(v_{1}, v_{2}\right)$ induced by vertices $v_{1}$ and $v_{2}$ is complete and $\Gamma_{v_{1}} \geq \Gamma_{v_{2}}$. If $G\left(v_{1}, v_{2}\right)$ is a maximal clique, then the algorithm terminates and outputs the maximal clique $G\left(v_{1}, v_{2}\right)$. Otherwise, the algorithm continues.

- General step. Having selected vertices $v_{1}, \ldots, v_{k}$ such that a subgraph $G\left(v_{1}, v_{2}, \ldots, v_{k}\right)$ induced by these vertices is complete and $\Gamma_{v_{1}} \geq \Gamma_{v_{2}} \geq$ $\ldots \geq \Gamma_{v_{k}}$, proceed as follows. If $G\left(v_{1}, v_{2}, \ldots, v_{k}\right)$ is a maximal clique, then the algorithm terminates and outputs the maximal clique $G\left(v_{1}, v_{2}, \ldots, v_{k}\right)$. If $G\left(v_{1}, v_{2}, \ldots, v_{k}\right)$ is not a maximal clique, then select a vertex $v_{k+1}$ such that $\Gamma_{v_{k+1}}=\max \left(\Gamma_{v}: v \sim v_{j}, j=1, \ldots, k\right)$. If there is more than one such vertex, then choose any of them arbitrary. In other words, at this step of the algorithm, we select a vertex $v_{k+1}$ such that $v_{k+1} \sim v_{j}, j=1, \ldots, k$, and $\Gamma_{v_{1}} \geq \Gamma_{v_{2}} \geq \ldots \geq \Gamma_{v_{k}} \geq \Gamma_{v_{k+1}}$. Having selected $v_{k+1}$ repeat the general step with complete subgraph $G\left(v_{1}, \ldots, v_{k}, v_{k+1}\right)$.

Definition 2.5. Given state $\mathbf{x} \in \mathbb{Z}_{+}^{V}$ with growth rates $\Gamma_{v}=\Gamma_{v}(\mathbf{x}), v \in V$, a maximal clique $G\left(v_{1}, \ldots, v_{m}\right)$ obtained by the algorithm above is called a final maximal clique for state $\mathbf{x}$.

Let $G\left(v_{1}, \ldots, v_{m}\right)$ be a final maximal clique for state $\mathbf{x}$. Then

$$
\begin{aligned}
& \Gamma_{v_{1}}=\max \left(\Gamma_{v}: v \in V\right) \\
& \Gamma_{v_{1}} \geq \ldots \geq \Gamma_{v_{m}} ; \\
& \Gamma_{v_{k+1}}=\max \left(\Gamma_{v}: v \sim v_{j}, j=1, \ldots, k\right), k=1, \ldots, m-1 .
\end{aligned}
$$

Example 2.6. Let $G$ be the graph in Figure 1.1. In this case, if the growth rates are such that vertices 5 and 6 are chosen at the first and the second step of the detection algorithm respectively, then the algorithm outputs final maximal clique $G(5,6,4)$.

Proposition 2.7. Let subgraph $G\left(v_{1}, \ldots, v_{m}\right)$ be a final maximal clique for state $\mathbf{x} \in \mathbb{Z}_{+}^{V}$ and let $D_{v_{i}}, i=1, \ldots, m$, be the corresponding D-sets. Let $\left(v_{k(1)}, \ldots, v_{k(n)}\right) \in$ $S(n)$ be such that $r$ particles are allocated at vertex $v_{k(n)}$ during the time interval $[1, n-1]$. Then

$$
\mathrm{P}_{\mathbf{x}}\left(A_{n}^{v_{k(n)}} \mid A_{n}^{V_{k(n)}}, \bigcap_{i=1}^{n-1} A_{i}^{v_{k(i)}}\right) \geq \frac{1}{1+|V| e^{-\alpha r}},
$$

where $|V|$ is the number of vertices of the graph $G=(V, E)$.

Proof of Proposition 2.\%. Observe that

$$
\mathrm{P}_{\mathbf{x}}\left(A_{n}^{v_{k(n)}} \mid A_{n}^{V_{k(n)}}, \bigcap_{i=1}^{n-1} A_{i}^{v_{k(i)}}\right)=\mathrm{P}_{\mathbf{y}}\left(A_{1}^{v_{k(n)}} \mid A_{1}^{V_{k(n)}}\right)
$$


where $\mathbf{y}=\mathbf{x}+\sum_{i=1}^{n-1} \mathbf{e}_{v_{k(i)}}$. If $D_{v_{k(n)}}=\emptyset$, then the conditional probability in (2.15) is trivially equal to 1 . Suppose that $D_{v_{k(n)}} \neq \emptyset$. Recall that, by assumption, there are $r$ particles at vertex $v_{k(n)}$ at time $n-1$. Therefore,

$$
\begin{aligned}
\Gamma_{v_{k(n)}}(\mathbf{y}) & =\Gamma_{v_{k(n)}}(\mathbf{x}) e^{\alpha r+\beta(n-1-r)}, \\
\Gamma_{v}(\mathbf{y}) & \leq \Gamma_{v}(\mathbf{x}) e^{\beta(n-1-r)}, \text { for } v \in D_{v_{k(n)}} .
\end{aligned}
$$

Consequently,

$$
\begin{aligned}
\mathrm{P}_{\mathbf{y}}\left(A_{1}^{v_{k(n)}} \mid A_{1}^{V_{k(n)}}\right) & \geq \frac{\Gamma_{v_{k(n)}}(\mathbf{x}) e^{\alpha r+\beta(n-1-r)}}{\Gamma_{v_{k(n)}}(\mathbf{x}) e^{\alpha r+\beta(n-1-r)}+e^{\beta(n-1-r)} \sum_{v \in D_{v_{k(n)}}} \Gamma_{v}(\mathbf{x})} \\
& =\frac{1}{1+e^{-\alpha r} \sum_{v \in D_{v_{k(n)}}} \frac{\Gamma_{v}(\mathbf{x})}{\Gamma_{v_{k(n)}}(\mathbf{x})}}
\end{aligned}
$$

By assumption, the subgraph $\left(v_{1}, \ldots, v_{m}\right)$ is a final maximal clique for the state $\mathbf{x}$. This implies that $\Gamma_{v_{k(n)}}(\mathbf{x}) \geq \Gamma_{v}(\mathbf{x})$ for $v \in D_{v_{k(n)}}$ and, hence, $\sum_{v \in D_{v_{k(n)}}} \frac{\Gamma_{v}(\mathbf{x})}{\Gamma_{v_{k(n)}}(\mathbf{x})} \leq$ $|V|$. Finally, we obtain that

$$
\mathrm{P}_{\mathbf{y}}\left(A_{1}^{v_{k(n)}} \mid A_{1}^{V_{k(n)}}\right) \geq \frac{1}{1+|V| e^{-\alpha r}},
$$

as claimed.

\section{Proof of Theorem 1.3}

3.1. Localisation in a final maximal clique. Define the following events.

$$
\begin{aligned}
& A_{n}^{\left(v_{1}, \ldots, v_{m}\right)}=\left\{\text { at time } n \text { a particle is placed at site } v \in\left(v_{1}, \ldots, v_{m}\right)\right\}, n \in \mathbb{Z}_{+}, \\
& A_{[1, n]}^{\left(v_{1}, \ldots, v_{m}\right)}=\bigcap_{k=1}^{n} A_{k}^{\left(v_{1}, \ldots, v_{m}\right)}, n \in \mathbb{Z}_{+} \cup\{\infty\} .
\end{aligned}
$$

Lemma 3.1. Let $X(n)=\left(X_{v}(n), v \in V\right)$ be a growth process with parameters $(\alpha, \beta)$ on a finite connected graph $G=(V, E)$ with at least two vertices. Given a state $\mathbf{x} \in \mathbb{Z}_{+}^{V}$ let a subgraph $G\left(v_{1}, \ldots, v_{m}\right)$ be a final maximal clique for the state $\mathbf{x}$, and let $0<\alpha \leq \beta$. Then there exists $\varepsilon>0$ depending only on $\alpha$ and the number of the graph vertices such that

$$
\mathrm{P}_{\mathbf{x}}\left(A_{[1, \infty]}^{\left(v_{1}, \ldots, v_{m}\right)}\right) \geq \varepsilon
$$

In other words, all particles can be allocated at vertices of a final maximal clique with probability that is not less than some $\varepsilon>0$ not depending on the initial state.

Proof of Lemma 3.1: It is easy to see that

$$
A_{[1, n]}^{\left(v_{1}, \ldots, v_{m}\right)}=\bigcup_{\left(v_{k(1)}, \ldots, v_{k(n)}\right) \in S(n)}\left(\bigcap_{i=1}^{n} A_{i}^{v_{k(i)}}\right),
$$

where events $A_{i}^{v_{k(i)}}$ are defined in (2.5), $S(n)$ is the set of sequences defined in (2.7), and the union is taken over all elements of $S(n)$. Therefore

$$
\mathrm{P}_{\mathbf{x}}\left(A_{[1, n]}^{\left(v_{1}, \ldots, v_{m}\right)}\right)=\sum_{\left(v_{k(1)}, \ldots, v_{k(n)}\right) \in S(n)} \mathrm{P}_{\mathbf{x}}\left(\bigcap_{i=1}^{n} A_{i}^{v_{k(i)}}\right) .
$$


Next, given $\left(v_{k(1)}, \ldots, v_{k(n)}\right) \in S(n)$ we are going to obtain a lower bound for the probability $\mathrm{P}_{\mathbf{x}}\left(\bigcap_{i=1}^{n} A_{i}^{v_{k(i)}}\right)$. Noting that $A_{i}^{v_{k}} \cap A_{i}^{V_{j}}=\emptyset$ for $k \neq j$ and recalling equation (2.8) we obtain that

$$
\begin{aligned}
& \mathrm{P}_{\mathbf{x}}\left(A_{n}^{v_{k(n)}} \mid \bigcap_{i=1}^{n-1} A_{i}^{v_{k(i)}}\right) \\
& =\mathrm{P}_{\mathbf{x}}\left(A_{n}^{v_{k(n)}} \mid A_{n}^{V_{k(n)}}, \bigcap_{i=1}^{n-1} A_{i}^{v_{k(i)}}\right) \mathrm{P}_{\mathbf{x}}\left(A_{n}^{V_{k(n)}} \mid \bigcap_{i=1}^{n-1} A_{i}^{v_{k(i)}}\right) \\
& =\mathrm{P}_{\mathbf{x}}\left(A_{n}^{v_{k(n)}} \mid A_{n}^{V_{k(n)}}, \bigcap_{i=1}^{n-1} A_{i}^{v_{k(i)}}\right) \mathrm{P}_{\mathbf{x}+\sum_{i=1}^{n-1} \mathbf{e}_{v_{k(i)}}}\left(A_{1}^{V_{k(n)}}\right) .
\end{aligned}
$$

Suppose that $X_{v_{k(n)}}(n-1)=X_{v_{k(n)}}(0)+r$ for some $0 \leq r \leq n-1$. In other words, $r$ particles are allocated at vertex $v_{k(n)}$ during the time interval $[1, n-1]$. Then, by Proposition 2.7,

$$
\mathrm{P}_{\mathbf{x}}\left(A_{n}^{v_{k(n)}} \mid A_{n}^{V_{k(n)}}, \bigcap_{i=1}^{n-1} A_{i}^{v_{k(i)}}\right) \geq \frac{1}{1+|V| e^{-\alpha r}} .
$$

Combining (3.5) and (3.6) gives that

$$
\mathrm{P}_{\mathbf{x}}\left(A_{n}^{v_{k(n)}} \mid \bigcap_{i=1}^{n-1} A_{i}^{v_{k(i)}}\right) \geq \frac{1}{1+|V| e^{-\alpha r}} \mathrm{P}_{\mathbf{x}+\sum_{i=1}^{n-1} \mathbf{e}_{v_{k(i)}}}\left(A_{1}^{V_{k(n)}}\right) .
$$

Consequently,

$$
\begin{aligned}
\mathrm{P}_{\mathbf{x}}\left(\bigcap_{i=1}^{n} A_{i}^{v_{k(i)}}\right) & =\mathrm{P}_{\mathbf{x}}\left(A_{n}^{v_{k(n)}} \mid \bigcap_{i=1}^{n-1} A_{i}^{v_{k(i)}}\right) \mathrm{P}_{\mathbf{x}}\left(\bigcap_{i=1}^{n-1} A_{i}^{v_{k(i)}}\right) \\
& \geq \frac{1}{1+|V| e^{-\alpha r}} \mathrm{P}_{\mathbf{x}+\sum_{i=1}^{n-1} \mathbf{e}_{v_{k(i)}}}\left(A_{1}^{V_{k(n)}}\right) \mathrm{P}_{\mathbf{x}}\left(\bigcap_{i=1}^{n-1} A_{i}^{v_{k(i)}}\right)
\end{aligned}
$$

Suppose that $\left(v_{k(1)}, \ldots, v_{k(n)}\right)$ is such that $n_{i}$ out of first $n$ particles are allocated at vertex $v_{i}, i=1, . ., m$, where $n_{1}, \ldots, n_{m}: n_{1}+\ldots+n_{m}=n$. Then, iterating equation (3.8) gives the following lower bound

$$
\mathrm{P}_{\mathbf{x}}\left(\bigcap_{i=1}^{n} A_{i}^{v_{k(i)}}\right) \geq \prod_{i=1}^{m}\left(\prod_{r=1}^{n_{i}-1} \frac{1}{1+|V| e^{-\alpha r}}\right) \mathbf{Q}_{\mathbf{x}, n}\left(\left(v_{k(1)}, \ldots, v_{k(n)}\right)\right),
$$

where probability $\mathbf{Q}_{\mathbf{x}, n}$ is defined in (2.9). It is easy to see that

$$
\mathrm{P}_{\mathbf{x}}\left(\bigcap_{i=1}^{n} A_{i}^{v_{k(i)}}\right) \geq \varepsilon_{n} \mathrm{Q}_{\mathbf{x}, n}\left(\left(v_{k(1)}, \ldots, v_{k(n)}\right)\right)
$$

where

$$
\varepsilon_{n}:=\left(\prod_{r=1}^{n-1} \frac{1}{1+|V| e^{-\alpha r}}\right)^{m} \geq\left(\prod_{r=1}^{\infty} \frac{1}{1+|V| e^{-\alpha r}}\right)^{m}=: \varepsilon>0 .
$$


Therefore, for every $\left(v_{k(1)}, \ldots, v_{k(n)}\right) \in S(n)$ we have that

$$
\mathrm{P}_{\mathbf{x}}\left(\bigcap_{i=1}^{n} A_{i}^{v_{k(i)}}\right) \geq \varepsilon \mathrm{Q}_{\mathbf{x}, n}\left(\left(v_{k(1)}, \ldots, v_{k(n)}\right)\right)
$$

Combining the preceding display with the fact that $\mathbf{Q}_{\mathbf{x}, n}$ is a probability measure on $S(n)$ (Proposition 2.4) gives that

$$
\mathrm{P}_{\mathbf{x}}\left(A_{[1, n]}^{\left(v_{1}, \ldots, v_{m}\right)}\right) \geq \varepsilon \sum_{\left(v_{k(1)}, \ldots, v_{k(n)}\right) \in S(n)} \mathrm{Q}_{\mathbf{x}, n}\left(\left(v_{k(1)}, \ldots, v_{k(n)}\right)\right)=\varepsilon
$$

Consequently, $\mathrm{P}_{\mathbf{x}}\left(A_{[1, \infty]}^{\left(v_{1}, \ldots, v_{m}\right)}\right) \geq \varepsilon$, where $\varepsilon>0$ (defined in (3.11)) does not depend on $\mathbf{x}$. The lemma is proved.

3.2. Eventual localisation. Let us show that, with probability one, the growth process eventually localises at a random maximal clique, as claimed. To this end, we use the renewal argument from the proof of Costa et al. (2018, Theorem 1). Given an arbitrary initial state $X(0)=\left(X_{v}(0), v \in V\right) \in \mathbb{Z}_{+}^{V}$ define the following sequence of random times $T_{k}, k \geq 0$. Set $T_{0}=0$. Suppose that time moments $T_{1}, \ldots, T_{k}$ are defined. Then, given a process state $X\left(T_{k}\right)$ at time $t=T_{k}$ let $G_{k}$ be a final maximal clique corresponding to state $X\left(T_{k}\right)$. Define $T_{k+1}$ as the first time moment when a particle is allocated in a vertex not belonging to $G_{k}$. By Lemma $3.1 \mathrm{P}\left(T_{k+1}<\infty \mid X\left(T_{k}\right)\right) \leq 1-\varepsilon$ for some $\varepsilon>0$. This yields that with probability one only a finite number of events $\left\{T_{k}<\infty\right\}$ occur. In other words, with probability one, eventually the growth process localises at a random maximal clique, as claimed.

3.3. Convergence of ratios $X_{v}(n) / X_{u}(n)$. Next we are going to show that if all particles are allocated at vertices of a maximal clique, then pairwise ratios $X_{v}(n) / X_{u}(n)$, where $v, u$ are any two vertices of the maximal clique, must converge, as claimed in Theorem 1.3. There are two cases to consider.

3.3.1. Case: $\alpha=\beta$. Let $\lambda:=\alpha=\beta$. Given state $\mathbf{x}=\left(x_{v}, v \in V\right) \in \mathbb{Z}_{+}^{V}$ let an induced subgraph $G\left(v_{1}, \ldots, v_{m}\right)$ be a final maximal clique for state $\mathbf{x}$. Define

$$
p_{i}:=\frac{\Gamma_{v_{i}}(\mathbf{x})}{\sum_{j=1}^{m} \Gamma_{v_{j}}(\mathbf{x})}, \quad i=1, \ldots, m .
$$

Given $\delta>0$ define the following subset of trajectories of the growth process

$$
B_{\delta}=\left\{\sum_{i=1}^{m}\left|X_{v_{i}}(n)-p_{i} n\right| \geq \delta n \text { for infinitely many } n\right\}
$$

and let $B_{\delta}^{c}$ be the complement of $B_{\delta}$. Then

$$
\mathrm{P}_{\mathbf{x}}\left(A_{[1, \infty]}^{\left(v_{1}, \ldots, v_{m}\right)}\right)=\mathrm{P}_{\mathbf{x}}\left(B_{\delta}^{c} \bigcap A_{[1, \infty]}^{\left(v_{1}, \ldots, v_{m}\right)}\right)+\mathrm{P}_{\mathbf{x}}\left(B_{\delta} \bigcap A_{[1, \infty]}^{\left(v_{1}, \ldots, v_{m}\right)}\right) .
$$

Proposition 3.2. For every $\delta>0$ and $\mathbf{x} \in \mathbb{Z}_{+}^{V}$

$$
\mathrm{P}_{\mathbf{x}}\left(B_{\delta} \bigcap A_{[1, \infty]}^{\left(v_{1}, \ldots, v_{m}\right)}\right)=0 .
$$


Proof of Proposition 3.2: Let $\left(v_{k(1)}, \ldots, v_{k(n)}\right) \in S(n)$. Observe that the assumption $\alpha=\beta$ implies the following equation

$$
\mathrm{P}_{\mathbf{x}}\left(A_{n}^{v_{k(n)}} \mid A_{n}^{\left(v_{1}, \ldots v_{m}\right)}, \bigcap_{i=1}^{n-1} A_{i}^{v_{k(i)}}\right)=p_{k(n)},
$$

where probabilities $p_{i}, i=1, \ldots, m$, are defined in (3.12). Therefore,

$$
\begin{aligned}
& \mathrm{P}_{\mathbf{x}}\left(A_{n}^{v_{k(n)}} \mid \bigcap_{i=1}^{n-1} A_{i}^{v_{k(i)}}\right) \\
& =\mathrm{P}_{\mathbf{x}}\left(A_{n}^{v_{k(n)}} \mid A_{n}^{\left(v_{1}, \ldots v_{m}\right)}, \bigcap_{i=1}^{n-1} A_{i}^{\left.v_{k(i)}\right)}\right) \mathrm{P}_{\mathbf{x}}\left(A_{n}^{\left(v_{1}, \ldots v_{m}\right)} \mid \bigcap_{i=1}^{n-1} A_{i}^{v_{k(i)}}\right) \\
& \leq \mathrm{P}_{\mathbf{x}}\left(A_{n}^{v_{k(n)}} \mid A_{n}^{\left(v_{1}, \ldots v_{m}\right)}, \bigcap_{i=1}^{n-1} A_{i}^{v_{k(i)}}\right)=p_{k(n)},
\end{aligned}
$$

and, hence,

$$
\begin{aligned}
\mathrm{P}_{\mathbf{x}}\left(\bigcap_{i=1}^{n} A_{i}^{v_{k(i)}}\right) & =\mathrm{P}_{\mathbf{x}}\left(A_{n}^{v_{k(n)}}\left(\bigcap_{i=1}^{n-1} A_{i}^{v_{k(i)}}\right) \mathrm{P}_{\mathbf{x}}\left(\bigcap_{i=1}^{n-1} A_{i}^{v_{k(i)}}\right)\right. \\
& \leq p_{k(n)} \mathrm{P}_{\mathbf{x}}\left(\bigcap_{i=1}^{n-1} A_{i}^{v_{k(i)}}\right) .
\end{aligned}
$$

Let $\left(v_{k(1)}, \ldots, v_{k(n)}\right)$ be such that

$$
\sum_{j=1}^{n} \mathbf{e}_{v_{k(j)}}=\sum_{k=1}^{m} n_{k} \mathbf{e}_{v_{k}}, \text { where } \sum_{k=1}^{m} n_{k}=n,
$$

i.e., $n_{i}$ out of first $n$ particles are allocated at vertex $v_{i}$. Then, iterating equation (3.16) gives the following upper bound for the probability of a fixed path of length $n$ of the growth process

$$
\mathrm{P}_{\mathbf{x}}\left(\bigcap_{j=1}^{n} A_{j}^{v_{k(j)}}\right) \leq p_{1}^{n_{1}} \cdots p_{m}^{n_{m}}
$$

Consider a random process $Y(n)=\left(Y_{1}(n), \ldots, Y_{m}(n)\right)$ describing results of independent trials, where in each trial a particle is allocated in one of $m$ boxes labeled by $i=1, \ldots, m$ with respective probabilities $p_{i}, i=1, \ldots, m$, and $Y_{i}(n)$ is the number of particles in box $i$ after $n$ trials. Let $\widetilde{\mathrm{P}}$ denote distribution of this process. It is easy to see that the right hand side of equation (3.17) is equal to probability $\widetilde{\mathrm{P}}\left(Y_{i}(n)=r_{i}, i=1, \ldots, m\right)$, computed given that the boxes are initially empty. Define

$$
\widetilde{B}_{\delta}=\left\{\sum_{i=1}^{m}\left|Y_{i}(n)-p_{i} n\right| \geq \delta n \text { for infinitely many } n\right\} .
$$

Equation (3.17) implies that $\mathrm{P}_{\mathbf{x}}\left(B_{\delta} \cap A_{[1, \infty]}^{\left(v_{1}, \ldots, v_{m}\right)}\right) \leq \widetilde{\mathrm{P}}\left(\widetilde{B}_{\delta}\right)$. By the strong law of large numbers for the i.i.d. case we have that $\widetilde{\mathrm{P}}\left(\widetilde{B}_{\delta}\right)=0$, and, hence,

$$
\mathrm{P}_{\mathbf{x}}\left(B_{\delta} \bigcap A_{[1, \infty]}^{\left(v_{1}, \ldots, v_{m}\right)}\right)=0
$$


as claimed.

It follows from Proposition 3.2 and equation (3.14) that

$$
\mathrm{P}_{\mathbf{x}}\left(\frac{X_{v_{i}}(n)}{X_{v_{j}}(n)} \rightarrow \frac{p_{i}}{p_{j}}, \text { as } n \rightarrow \infty \mid A_{[1, \infty]}^{\left(v_{1}, \ldots, v_{m}\right)}\right)=1,
$$

for $i, j=1, \ldots, m$. Finally, a direct computation gives that $\frac{p_{i}}{p_{j}}=\frac{\Gamma_{v_{i}}(\mathbf{x})}{\Gamma_{v_{j}}(\mathbf{x})}=e^{C_{v_{i} v_{j}}}$, where

$$
C_{v_{i} v_{j}}=\lambda \lim _{n \rightarrow \infty} \sum_{w \in V} X_{w}(n)\left[\mathbf{1}_{\left\{w \sim v_{i}, w \nsim v_{j}\right\}}-\mathbf{1}_{\left\{w \nsim v_{i}, w \sim v_{j}\right\}}\right], \text { for } i, j=1, \ldots, m .
$$

The proof of Theorem 1.3 in the case $\alpha=\beta$ is now finished.

3.3.2. Case: $\alpha<\beta$. We start with an auxiliary statement (Lemma 3.3) that might be of interest on its own right.

Lemma 3.3. Let $X(n)=\left(X_{1}(n), \ldots, X_{m}(n)\right)$ be a growth process with parameters $0<\alpha<\beta$ on a complete graph with $m \geq 2$ vertices labeled by $1, \ldots, m$, and let $Z_{i}(n)=X_{i}(n)-X_{m}(n), i=1, \ldots, m-1$. Then $Z(n):=\left(Z_{1}(n), \ldots, Z_{m-1}(n)\right) \in$ $\mathbb{Z}^{m-1}$ is an irreducible positive recurrent Markov chain.

Proof of Lemma 3.3: Let $X(0)=\mathbf{x}=\left(x_{1}, \ldots, x_{m}\right) \in \mathbb{Z}_{+}^{m}$. For short, denote $\Gamma_{i}=$ $\Gamma_{i}(\mathbf{x}), i=1, \ldots, m$, and $-\lambda=\alpha-\beta<0$. Note that if $\mathbf{y}=\left(x_{1}+r_{1}, \ldots, x_{m}+r_{m}\right) \in$ $\mathbb{Z}_{+}^{m}$, where $\sum_{i=1}^{m} r_{i}=n$, then

$$
\Gamma_{i}(\mathbf{y})=\Gamma_{i} e^{\alpha r_{i}+\beta\left(n-r_{i}\right)}=\Gamma_{i} e^{-\lambda r_{i}} e^{\beta n}, \quad i=1, \ldots, m .
$$

Therefore

$$
\mathrm{P}\left(Z(n+1)=Z(n)+\mathbf{e}_{i} \mid Z(n)=\mathbf{z}\right)=\frac{\Gamma_{i} e^{-\lambda z_{i}}}{\Gamma_{m}+\sum_{i=1}^{m-1} \Gamma_{i} e^{-\lambda z_{i}}}
$$

for $i=1, \ldots, m-1$, and

$$
\mathrm{P}(Z(n+1)=Z(n)-\mathbf{e} \mid Z(n)=\mathbf{z})=\frac{\Gamma_{m}}{\Gamma_{m}+\sum_{i=1}^{m-1} \Gamma_{i} e^{-\lambda z_{i}}},
$$

for all $\mathbf{z}=\left(z_{1}, \ldots, z_{m-1}\right) \in \mathbb{Z}^{m-1}$, where $\mathbf{e}_{i}$ is now the $i$-th unit vector in $\mathbb{Z}^{m-1}$, and $\mathbf{e}=\mathbf{e}_{1}+\cdots+\mathbf{e}_{m-1} \in \mathbb{Z}^{m-1}$. Thus, $Z(n)$ is a Markov chain with transition probabilities given by (3.18)-(3.19). It is easy to see that this Markov chain is irreducible. Further, define the following function

$$
f(\mathbf{z})=\sum_{i=1}^{m-1} z_{i}^{2}, \quad \mathbf{z}=\left(z_{1}, \ldots, z_{m-1}\right) \in \mathbb{Z}^{m-1},
$$

and show that given $\varepsilon>0$

$$
\begin{aligned}
\mathrm{E}(f(Z(n+1))- & f(Z(n)) \mid Z(n)=\mathbf{z}) \leq-\varepsilon, \\
\quad \text { for } \mathbf{z} & =\left(z_{1}, \ldots, z_{m-1}\right) \in \mathbb{Z}_{+}^{m-1}:\left|z_{1}\right|+\ldots+\left|z_{m-1}\right| \geq C,
\end{aligned}
$$

provided that $C=C(\varepsilon)>0$ is sufficiently large. Indeed, fix $\varepsilon>0$. A direct computation gives that

$$
\mathrm{E}(f(Z(n+1))-f(Z(n)) \mid Z(n)=\mathbf{z})+\varepsilon=\frac{\varepsilon+\sum_{i=1}^{m-1} h_{i}\left(z_{i}, \varepsilon\right)}{W(\mathbf{z})},
$$


where $h_{i}(z, \varepsilon):=(2 z+1+\varepsilon) a_{i} e^{-\lambda z}-2 z+1$ for $z \in \mathbb{R}, a_{i}=\frac{\Gamma_{i}}{\Gamma_{m}}, i=1, \ldots, m-1$, and $W(\mathbf{z})=1+\sum_{i=1}^{m-1} a_{i} e^{-\lambda z_{i}}$. It is easy to see that for each $i=1, \ldots, m-1$, there exists $C_{i}>0$ such that $h_{i}(z, \varepsilon) \leq-|z|$ for $|z|>C_{i}$. Define

$$
H(\varepsilon):=\max _{i=1, \ldots, m-1} \sup _{-\infty<z<\infty}\left(h_{i}(z, \varepsilon)+|z|\right) .
$$

Note that $H(\varepsilon)>0$, as $h_{i}(0, \varepsilon)=a_{i}+1+\varepsilon>0, i=1, \ldots, m-1$. It follows from the definition of $H$ that

$$
\sum_{i=1}^{m-1} h_{i}\left(z_{i}, \varepsilon\right)=\sum_{i=1}^{m-1}\left(h_{i}\left(z_{i}, \varepsilon\right)+\left|z_{i}\right|\right)-\left|z_{i}\right| \leq(m-1) H(\varepsilon)-\sum_{i=1}^{m-1}\left|z_{i}\right| .
$$

Combining the preceding equation with equation (3.22) gives equation (3.21), where $C=\varepsilon+(m-1) H(\varepsilon)$. Thus, positive recurrence of Markov chain $Z(n)$ follows from the Foster criterion for positive recurrence of a Markov chain (e.g. Menshikov et al., 2017, Theorem 2.6.4) with the Lyapunov function $f$.

Remark 3.4. Note that Lemma 3.3 is reminiscent of Shcherbakov and Volkov (2010b, Theorem 1, Part (1)). Moreover, to show positive recurrence of the Markov chain $Z(n)$ we use the criterion for positive recurrence with the same Lyapunov function (3.20) as in the proof of positive recurrence of a similar Markov chain in Shcherbakov and Volkov (2010b, Theorem 1, Part (1)).

The next step of the proof is to show the convergence of the ratios in the case of a complete graph. This is the subject of the following lemma.

Lemma 3.5 (The strong law of large numbers for a growth process on a complete graph). Let $X(n)=\left(X_{1}(n), \ldots, X_{m}(n)\right)$ be a growth process with parameters $0<\alpha<\beta$ on a complete graph with $m \geq 1$ vertices labeled by $1, \ldots, m$. For every initial state $X(0)=\mathbf{x} \in \mathbb{Z}_{+}^{m}$ and $\delta>0$ with probability one

$$
\sum_{i=1}^{m}\left|X_{i}(n)-\frac{n}{m}\right|>n \delta \text { for finitely many } n \text {. }
$$

In other words, with probability one $\lim _{n \rightarrow \infty} \frac{X_{i}(n)}{n}=\frac{1}{m}, i=1, \ldots, m$.

Proof of Lemma 3.5: Note that if $\sum_{i=1}^{m}\left|X_{i}(n)-n / m\right|>n \delta$, then $\sum_{i=1}^{m-1}\left|Z_{i}(n)\right|>$ $n \delta / m^{2}$, where $Z(n)$ is the Markov chain defined in Lemma 3.3. Therefore, to prove the lemma it suffices to show that, given $\delta^{\prime}>0$ with probability one, only a finite number of events $\sum_{i=1}^{m-1}\left|Z_{i}(n)\right|>n \delta^{\prime}$ occurs.

Let $\sigma_{0}=0$ and let $\sigma_{k}=\inf \left(n>\sigma_{k-1}: Z(n)=\mathbf{0}\right)$ for $k \geq 1$. In other words, $\sigma_{k}$ is the $k$-th return time to the origin for the Markov chain $Z(n)$. Define the following events

$$
W_{k, \delta^{\prime}}:=\left\{\max _{n \in\left(\sigma_{k}, \sigma_{k+1}\right)} \sum_{i=1}^{m-1}\left|Z_{i}(n)\right|>n \delta^{\prime}\right\}, k \geq 1 .
$$

Note that $\sum_{i=1}^{m-1}\left|Z_{i}(n)\right|$ can increase at most by $(m-1)$ at each time step, and, besides, $\sigma_{k} \geq k$. This yields that

$$
W_{k, \delta^{\prime}} \subseteq\left\{\sigma_{k+1}-\sigma_{k} \geq \frac{k \delta^{\prime}}{m-1}\right\}
$$


Assume, without loss of generality, that $Z(0)=\mathbf{0}$. Then random variables $\sigma_{k}-$ $\sigma_{k-1}, k \geq 1$, are identically distributed with the same distribution as the first return $\sigma_{1}$. It follows from Lemma 3.3 that $\mathrm{E}\left(\sigma_{1}\right)<\infty$. Therefore,

$$
\begin{aligned}
\sum_{k=1}^{\infty} \mathrm{P}\left(\sigma_{k}-\sigma_{k-1} \geq k \delta^{\prime} /(m-1) \mid Z(0)=\mathbf{0}\right) & =\sum_{k=1}^{\infty} \mathrm{P}\left(\sigma_{1} \geq k \delta^{\prime} /(m-1) \mid Z(0)=\mathbf{0}\right) \\
& \leq C \mathrm{E}\left(\sigma_{1} \mid Z(0)=\mathbf{0}\right)<\infty
\end{aligned}
$$

and, hence, by the Borel-Cantelli lemma, with probability one, only a finite number of events $\left\{\sigma_{k}-\sigma_{k-1} \geq k \delta^{\prime} /(m-1)\right\}, k \geq 1$, occur. Recalling equation (3.24) gives that, with probability one, only a finite number of events $W_{k, \delta^{\prime}}$ occur. Consequently, with probability one, $\sum_{i=1}^{m-1}\left|Z_{i}(n)\right|>n \delta^{\prime}$ only for finitely many $n$, and the lemma is proved.

Finally, we are going to show the convergence of the ratios for the growth process with parameters $0<\alpha<\beta$ on an arbitrary connected graph $G(V, E)$. Let $\left(v_{1}, \ldots, v_{m}\right) \subseteq V$ be vertices of a clique. Fix $\left(v_{k(1)}, \ldots, v_{k(n)}\right) \in S(n)$. A direct computation gives the following analogue of bound (3.15)

$$
\begin{aligned}
\mathrm{P}_{\mathbf{x}}\left(A_{1}^{v_{k(1)}}\right) & =\mathrm{P}_{\mathbf{x}}\left(A_{1}^{v_{k(1)}} \mid A_{1}^{\left(v_{1}, \ldots v_{m}\right)}\right) \mathrm{P}_{\mathbf{x}}\left(A_{1}^{\left(v_{1}, \ldots v_{m}\right)}\right) \\
& \leq \mathrm{P}_{\mathbf{x}}\left(A_{1}^{v_{k(1)}} \mid A_{1}^{\left(v_{1}, \ldots v_{m}\right)}\right)=\frac{\Gamma_{v_{k(1)}}}{\sum_{k=1}^{m} \Gamma_{v_{k}}},
\end{aligned}
$$

where, as before, we denoted $\Gamma_{v_{k}}=\Gamma_{v_{k}}(\mathbf{x}), k=1, \ldots, m$. Similarly, we have for every $j=2, \ldots, n$ that

$$
\begin{aligned}
& \mathrm{P}_{\mathbf{x}}\left(A_{j}^{v_{k(j)}} \mid \bigcap_{i=1}^{j-1} A_{i}^{v_{k(i)}}\right) \\
& =\mathrm{P}_{\mathbf{x}}\left(A_{j}^{v_{k(j)}} \mid A_{j}^{\left(v_{1}, \ldots v_{m}\right)}, \bigcap_{i=1}^{j-1} A_{i}^{v_{k(i)}}\right) \mathrm{P}_{\mathbf{x}}\left(A_{j}^{\left(v_{1}, \ldots v_{m}\right)} \mid \bigcap_{i=1}^{j-1} A_{i}^{v_{k(i)}}\right) \\
& \leq \mathrm{P}_{\mathbf{x}}\left(A_{j}^{v_{k(j)}} \mid A_{j}^{\left(v_{1}, \ldots v_{m}\right)}, \bigcap_{i=1}^{j-1} A_{i}^{v_{k(i)}}\right)=\frac{\Gamma_{v_{k(j)}} e^{-\lambda r_{k(j), j-1}}}{\sum_{k=1}^{m} \Gamma_{v_{k}} e^{-\lambda r_{k, j-1}}},
\end{aligned}
$$

where $\lambda=-(\alpha-\beta)$ and $r_{k, j-1}, k=1, \ldots, m$, are such that

$$
\sum_{i=1}^{j-1} \mathbf{e}_{v_{k(i)}}=\sum_{k=1}^{m} r_{k, j-1} \mathbf{e}_{v_{k}} \text { for } j \geq 2 \text { and } r_{k, 0}=0 .
$$

In other words, $r_{k, j-1}$ is the number of particles at vertex $k$ at time $j-1$. Then, it follows from equations (3.25) and (3.26) that

$$
\mathrm{P}_{\mathbf{x}}\left(\bigcap_{i=1}^{n} A_{i}^{v_{k(i)}}\right) \leq \prod_{i=1}^{n} \frac{\Gamma_{v_{k(i)}} e^{-\lambda r_{k(i), i-1}}}{\sum_{k=1}^{m} \Gamma_{v_{k}} e^{-\lambda r_{k, i-1}}}
$$

Consider a growth process $\widetilde{X}(n)$ with parameters $(\alpha, \beta)$ on the complete graph with vertices $1, \ldots, m$, whose growth rates are computed as follows

$$
\Gamma_{i}(\widetilde{\mathbf{x}})=\Gamma_{v_{i}} e^{\alpha \tilde{x}_{i}+\beta \sum_{j \neq i} \tilde{x}_{j}}, \widetilde{\mathbf{x}}=\left(\tilde{x}_{1}, \ldots, \tilde{x}_{m}\right) \in \mathbb{Z}_{+}^{m},
$$

where, in contrast to growth rates (1.1), additional coefficients $\Gamma_{v_{i}}$ appear. Assume that $\tilde{X}(0)=\mathbf{0}$. Then, it is easy to see that the right-hand side of equation (3.27) is 
the probability of a trajectory of length $n$ of the growth process $\widetilde{X}(n)$ corresponding to the sequence $\left(v_{k(1)}, \ldots, v_{k(n)}\right) \in S(n)$ as follows. This is a trajectory such that a particle is allocated at vertex $k(i) \in(1, \ldots, m)$ at time $i=1, . ., n$. Further, given $\delta>0$ the following analogue of equation (3.14) holds

$$
\mathrm{P}_{\mathbf{x}}\left(A_{[1, \infty]}^{\left(v_{1}, \ldots, v_{m}\right)}\right)=\mathrm{P}_{\mathbf{x}}\left(B_{\delta}^{c} \bigcap A_{[1, \infty]}^{\left(v_{1}, \ldots, v_{m}\right)}\right)+\mathrm{P}_{\mathbf{x}}\left(B_{\delta} \bigcap A_{[1, \infty]}^{\left(v_{1}, \ldots, v_{m}\right)}\right)
$$

where now

$$
B_{\delta}=\left\{\sum_{i=1}^{m}\left|X_{v_{i}}(n)-\frac{n}{m}\right| \geq \delta n \text { for infinitely many } n\right\}
$$

and $B_{\delta}^{c}$ is, as before, the complement of $B_{\delta}$. It follows from equation (3.27) that

$$
\mathrm{P}_{\mathbf{x}}\left(B_{\delta} \bigcap A_{[1, \infty]}^{\left(v_{1}, \ldots, v_{m}\right)}\right) \leq \widetilde{\mathrm{P}}\left(\widetilde{B}_{\delta}\right)
$$

where $\widetilde{\mathrm{P}}$ is the distribution of the growth process $\widetilde{X}(n)$ on the complete graph with $m$ vertices (with growth rates (3.28)) starting at $\widetilde{X}(0)=\mathbf{0} \in \mathbb{Z}_{+}^{m}$ and

$$
\widetilde{B}_{\delta}=\left\{\sum_{i=1}^{m}\left|\widetilde{X}_{v_{i}}(n)-\frac{n}{m}\right| \geq \delta n \text { for infinitely many } n\right\} .
$$

Note that both Lemma 3.3 and Lemma 3.5 remain true for this growth process (the proofs can be repeated verbatim). Therefore, $\mathrm{P}\left(\widetilde{B}_{\delta}\right)=0$, and, hence, $\mathrm{P}_{\mathbf{x}}\left(B_{\delta} \bigcap A_{[1, \infty]}^{\left(v_{1}, \ldots, v_{m}\right)}\right)=0$. This yields that

$$
\mathrm{P}_{\mathbf{x}}\left(\frac{X_{v_{i}}(n)}{X_{v_{j}}(n)} \rightarrow 1, \text { as } n \rightarrow \infty \mid A_{[1, \infty]}^{\left(v_{1}, \ldots, v_{m}\right)}\right)=1
$$

for $i, j=1, \ldots, m$, as claimed.

The proof of Theorem 1.3 in the case $0<\alpha<\beta$ is finished.

\section{Proof of Theorem 1.6}

Start with the following proposition.

Proposition 4.1. Let $X(n)=\left(X_{v}(n), v \in V\right)$ be a growth process with parameters $(\alpha, \beta)$ on a finite connected graph $G=(V, E)$ and let $0<\beta<\alpha$. Given state $\mathbf{x} \in \mathbb{Z}_{+}^{V}$ with growth rates $\Gamma_{v}(\mathbf{x}), v \in V$, suppose that $\Gamma_{u}(\mathbf{x})=\max \left(\Gamma_{v}(\mathbf{x}): v \in V\right)$. Then $\mathrm{P}_{\mathbf{x}}\left(A_{[1, \infty]}^{u}\right) \geq \varepsilon$ for some $\varepsilon>0$ that depends only on $\alpha, \beta$ and $|V|$. In other words, with positive probability, all subsequent particles will be allocated at a vertex with the maximal growth rate.

Proof of Proposition 4.1. The proof of the lemma is similar to the proof of Lemma 1 in Costa et al. (2018). We provide the details for the sake of completeness. Note that $\Gamma_{u}\left(\mathbf{x}+n \mathbf{e}_{u}\right)=\Gamma_{u}(\mathbf{x}) e^{\alpha n}$ and $\Gamma_{v}\left(\mathbf{x}+n \mathbf{e}_{u}\right) \leq \Gamma_{v}(\mathbf{x}) e^{\beta n}, v \neq u$. Therefore, using that $\Gamma_{u}(\mathbf{x}) \geq \Gamma_{v}(\mathbf{x})$ for $v \neq u$ we obtain that

$$
\sum_{v \neq u} \frac{\Gamma_{v}\left(\mathbf{x}+n \mathbf{e}_{u}\right)}{\Gamma_{u}\left(\mathbf{x}+n \mathbf{e}_{u}\right)} \leq|V| e^{-(\alpha-\beta) n}
$$


which, in turn, gives that

$$
\begin{aligned}
\mathrm{P}_{\mathbf{x}}\left(A_{[1, \infty]}^{u}\right) & =\prod_{n=0}^{\infty} \frac{\Gamma_{u}(\mathbf{x}) e^{\alpha n}}{\Gamma_{u}(\mathbf{x}) e^{\alpha n}+\sum_{v \neq u} \Gamma_{v}\left(\mathbf{x}+n \mathbf{e}_{u}\right)} \\
& \geq \prod_{n=0}^{\infty} \frac{1}{1+|V| e^{-(\alpha-\beta) n}}=: \varepsilon>0
\end{aligned}
$$

as claimed.

The proof of Theorem 1.6 can be finished by using the renewal argument similarly to the proof of Theorem 1.3. We omit the details.

\section{Acknowledgements}

We thank Stanislav Volkov and an anonymous referee for useful comments that improved the presentation.

\section{References}

Basdevant, A.-L., Schapira, B., and Singh, A. Localization on 4 sites for vertex-reinforced random walks on $\mathbb{Z}$. Ann. Probab., 42 (2), 527-558 (2014). MR3178466.

Benaïm, M., Benjamini, I., Chen, J., and Lima, Y. A generalized Pólya's urn with graph based interactions. Random Structures Algorithms, 46 (4), 614-634 (2015). MR3346459.

Costa, M., Menshikov, M., Shcherbakov, V., and Vachkovskaia, M. Localisation in a growth model with interaction. J. Stat. Phys., 171 (6), 1150-1175 (2018). MR3805592.

Davis, B. Reinforced random walk. Probab. Theory Related Fields, 84 (2), 203-229 (1990). MR1030727.

Evans, J. W. Random and cooperative sequential adsorption. Rev. Modern Phys., 65, 1281-1329 (1993). DOI: 0.1103/RevModPhys.65.1281.

Fleurke, S. R., Formentin, M., and Külske, C. Dependent particle deposition on a graph: concentration properties of the height profile. Markov Process. Related Fields, 17 (2), 187-208 (2011). MR2856239.

Janson, S., Shcherbakov, V., and Volkov, S. Long term behaviour of a reversible system of interacting random walks. J. Stat. Phys., 175 (1), 71-96 (2019). MR3927218.

Menshikov, M., Popov, S., and Wade, A. Non-homogeneous random walks. Lyapunov function methods for near-critical stochastic systems, volume 209 of Cambridge Tracts in Mathematics. Cambridge University Press, Cambridge (2017). ISBN 978-1-107-02669-8. MR3587911.

Shcherbakov, V. and Volkov, S. Queueing with neighbours. In Probability and mathematical genetics, volume 378 of London Math. Soc. Lecture Note Ser., pp. 464-482. Cambridge Univ. Press, Cambridge (2010a). MR2744252.

Shcherbakov, V. and Volkov, S. Stability of a growth process generated by monomer filling with nearest-neighbour cooperative effects. Stochastic Process. Appl., 120 (6), 926-948 (2010b). MR2610332.

Shcherbakov, V. and Volkov, S. Long term behaviour of locally interacting birthand-death processes. J. Stat. Phys., 158 (1), 132-157 (2015). MR3296278. 
Volkov, S. Vertex-reinforced random walk on arbitrary graphs. Ann. Probab., 29 (1), 66-91 (2001). MR1825142. 